\title{
L'UNION EUROPÉENNE ÉLARGIE ET SES VOISINS MÉDITERRANÉENS : LES PERSPECTIVES D'INTÉGRATION
}

\section{Sébastien Dupuch, El Mouhoub Mouhoud \& Fatiha Talahite ${ }^{1}$}

\author{
Date de réception de l'article: 11 juin 2003 \\ Date d'acceptation pour publication: 16 mars 2004
}

\begin{abstract}
RÉSUMÉ. La vision d'une Europe relativement homogène se modifie avec l'élargissement et le partenariat Euro-med. L'évaluation de l'insertion internationale des pays partenaires méditerranéens (PPM) montre que relativement aux nouveaux membres de I'Union élargie, les PPM connaissent une faible convergence réelle et structurelle. Les indices de Krugman et de FingerKreinin révèlent, qu'à l'exception de la Turquie, les pays méditerranéens sont complémentaires des pays de l'UE et demeurent très spécialisés dans des secteurs intensifs en main-d'œuvre ou en ressources naturelles. La faible efficacité des réformes structurelles et l'absence d'intégration Sud-Sud s'ajoutent aux insuffisances de l'accord de libre-échange lui-même. L'attractivité des PPM aux investissements directs étrangers demeure ainsi limitée alors que leur rôle est important pour leur rattrapage et le développement d'une spécialisation intra-branche.
\end{abstract}

\section{Classification JEL : F15, F21, 052, 055 \\ Mots-clés: Union européenne; partenariat euro-méditerranéen; spécialisation; investissements directs étrangers.}

ABStRACT. The concept of a quite homogeneous Europe has been changing with the enlargement and the Euromed partnership. An assessment of the international integration of Mediterranean Partner Countries (MPCs) shows that these countries experience weak convergence relative to new EU members. Krugman and Finger-Kreinin indices underline the fact that, with the exception of Turkey, MPCs have complementary trade structures relative to the EU and remain much specialised in labour and resource-intensive industries. Inefficient structural reforms, the lack of South-South integration and inherent shortcomings of the agreement limit the attractiveness of these countries to foreign investors, though they could contribute to their catching-up and the upgrading of their trade specialisation.

JEL Classification: F15; F21; 052; 055. Keywords: European Union; Euro-Mediterranean partnership; Specialisation; Foreign Direct Investment.

\footnotetext{
1. Auteur correspondant: El Mouhoub Mounoud, Professeur d'économie, Université Paris XIII et directeur du CEPNCNRS-UMR 7115 (mouhoud@seg.univ-paris13.fr) ;

Sébastien DuPUCH, Attaché Temporaire d'Enseignement et de Recherche (ATER) à I'Université Paris XIII, CEPN (Centre d'Économie de l'université Paris-Nord); Fatiha TALAHITE, Chargée de recherche CNRS, CEPN (Centre d'Économie de I'université Paris-Nord).
} 
Chaque nouvelle étape de l'unification européenne relance les interrogations relatives aux effets de l'intégration régionale sur les structures productives des pays membres. Aujourd'hui, l'élargissement aux pays d'Europe Centrale et Orientale (PECO) et le partenariat Euromediterranéen conduisent à réexaminer la configuration de la géographie économique européenne et de ses voisins.

Contrairement à l'expérience des États-Unis, les pays de I'Union européenne, à chaque nouvelle étape de l'intégration, ont développé des échanges commerciaux de type intra-branche, reflet d'une diversification de leurs activités économiques. Le rattrapage des nouveaux pays membres s'accompagne souvent, à l'exemple de l'Espagne, d'une transformation de la structure des échanges vers un schéma de spécialisation de type intra-branche, fondé sur des échanges de produits différenciés verticalement (qualités différentes) et dans une moindre mesure horizontalement (différentes variétés à qualité identique).

Cette transformation est liée en partie à la nature des investissements directs étrangers (IDE) réalisés dans le cadre de l'intégration à I'UE. Ces IDE s'effectuent dans des branches diversifiées au lieu de se cantonner dans les secteurs traditionnels et intensifs en travail peu qualifié. Les IDE concourent ainsi à modifier la spécialisation internationale des pays d'accueil (Hanaut et al., 2001). À cette convergence des structures de spécialisation s'ajoute un rapprochement du fonctionnement des marchés et des institutions dans leur ensemble. La politique de cohésion européenne (fonds de cohésion et fonds structurels) est supposée favoriser le rattrapage des régions retardataires en termes d'infrastructures et de développement. La conception d'ensemble développée dans les rapports qui ont servi à la préparation de l'achèvement du marché unique et de l'union monétaire est que les risques de chocs asymétriques sont faibles en présence d'échanges intra-branche dominants et ne justifient pas la mise en œuvre d'un véritable fédéralisme budgétaire (Emerson, 1990).

Un travail récent (Dupuch et al., 2001 ; 2004) a évalué les effets de l'élargissement de l'Union européenne aux pays d'Europe Centrale et Orientale (PECO) sur la spécialisation des activités. II en résulte que certains PECO avancés (République Tchèque, Slovénie, Hongrie) s'orientent vers le modèle espagnol de rattrapage et de diversification des activités et tendent vers le schéma européen de spécialisation intra-branche. À l'opposé, les autres pays risquent de rester enfermés dans une spécialisation inter-branche traditionnelle et de connaître une logique de type centre-périphérie vis-à-vis de I'UE. En outre, cet élargissement est susceptible de remettre en cause à moyen terme le schéma de diversification européenne. Les risques de décrochage de certains pays d'Europe du Sud (Grèce, Portugal) directement concurrencés par les PECO dans leurs principaux domaines ne sont pas en effet à négliger.

Cet article se propose d'étendre l'analyse au voisinage de I'UE dans le cadre du partenariat Euro-méditérranéen. Nous cherchons à évaluer, dans le cas des pays partenaires méditerranéens (PPM), la pertinence de chacun des deux scénarios de spécialisation inter et intrabranche. L'accord de libre-échange Euro-méditerranéen, bien que n'ayant ni la portée ni la couverture sectorielle de l'accord d'élargissement aux PECO, constitue une avancée dans 
I'intégration commerciale entre l'UE et les PPM, mais sa mise en œuvre est trop récente pour analyser, ex post, ses conséquences macro-économiques et ses effets sur la spécialisation internationale. En comparaison avec les PECO, dans quelles conditions les PPM ${ }^{2}$ s'insèrent-ils dans la géographie économique de l'Europe et quel schéma de spécialisation et de convergence tendent-ils à emprunter?

Dans un premier temps, il s'agit de rassembler les principaux faits stylisés sur les conditions d'insertion des PPM. Dans un second temps, on étudie le cadre institutionnel de l'accord de libre-échange entre I'UE et les PPM en relation avec les réformes structurelles entreprises dans ces pays. Enfin, I'évolution de la spécialisation des PPM est mesurée en comparaison avec les PECO et les pays d'Europe du Sud. Le rôle des IDE dans la transformation éventuelle de la spécialisation est également analysé.

\section{LES CONDITIONS D'INSERTION DES PPM À L'UNION EUROPÉENNE : UNE COMPARAISON avec les pays de l'Europe du Sud et les PECO}

Avant de s'intéresser au contenu de l'accord de libre-échange euro-méditerranéen et à la nature de la spécialisation internationale des PPM, il semble opportun de dresser un bilan de la convergence réelle et structurelle de ces pays par rapport aux PECO d'une part, et aux pays d'Europe du Sud d'autre part. Nous comparons ici l'évolution des principales variables de convergence réelle et structurelle entre les différentes zones périphériques de I'UE (PIB par tête, productivité, structures productives, technologie et capital humain...).

\section{Convergence des niveaux de vie par habitant: éléments de comparaison}

Par rapport à la France ${ }^{3}$, les PPM conservent une position structurellement faible en termes de PIB par tête. On distingue initialement deux groupes de pays: I'Algérie, la Turquie et la Tunisie présentent des PIB par habitant qui s'élèvent à environ $25 \%$ celui de la France alors que l'Égypte et le Maroc demeurent à des niveaux inférieurs à $15 \%$. En outre, ces pays connaissent une très faible convergence. L'Algérie connaît même une régression de dix points entre 1980 et 2003 alors qu'initialement elle se situait nettement au-dessus des autres PPM (GRAPHIQUE 1).

2. Parmi les douze pays partenaires méditerranéens, cet article ne traite que cinq d'entre eux: la Turquie, les trois pays du Maghreb et l'Égypte.

3. Pour mesurer la convergence des PIB par habitant, nous prenons la France comme référence au lieu de la moyenne de l'Union européenne, en raison de l'importance des échanges commerciaux des pays du Sud de la Méditerranée avec ce pays. La référence à la France offre en outre des avantages de stabilité dans la mesure où la composition des membres de l'UE s'est largement modifiée ces dernières années. 


\section{Graphique 1 - Convergence réelle* des $\mathrm{PPM}^{* *}$ par rapport à la France}

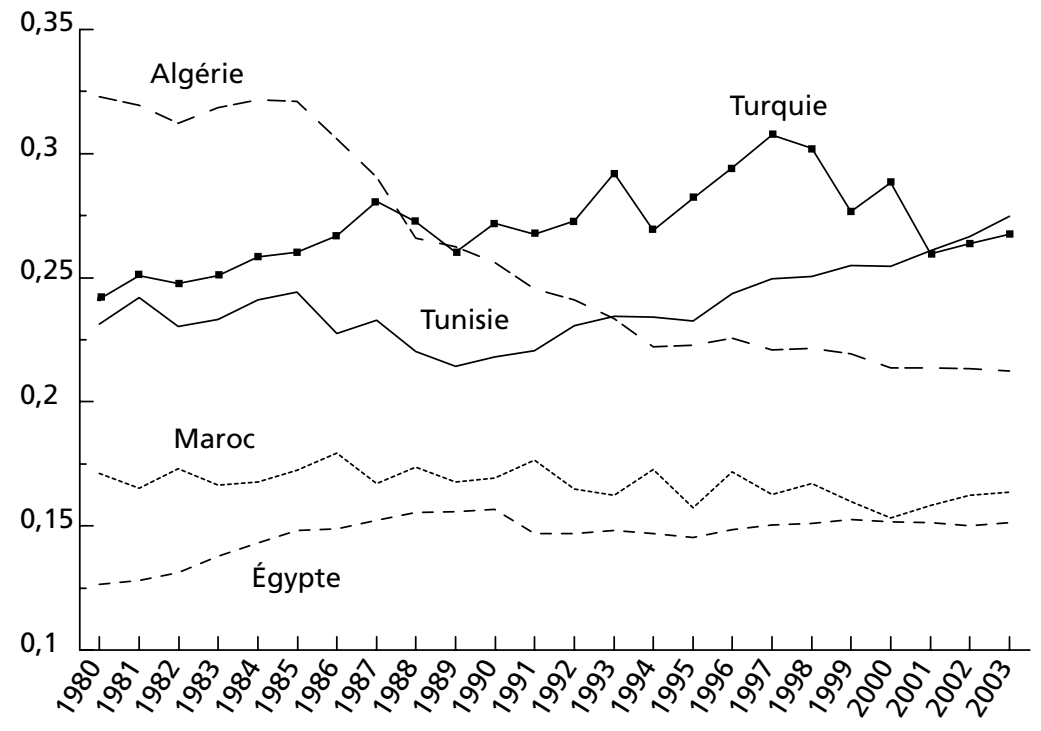

* PIB par habitant exprimé en parité de pouvoir d'achat, France $=1$.

** Pays partenaires méditerranéens.

Source: FMI, calculs des auteurs.

Les écarts entre les différentes zones périphériques sont relativement élevés. Les pays du Sud de l'Europe ont connu depuis leur adhésion une convergence réelle par rapport aux grands pays européens. Le processus semble néanmoins interrompu au Portugal qui tend de nouveau à diverger depuis la fin des années quatre-vingt-dix. Les pays candidats convergent également rapidement à l'exception de la Bulgarie et de la Roumanie dont l'écart avec la France reste stable sur la période. Ils présentent toutefois des PIB par habitant très inférieurs à ceux des pays d'Europe du Sud lorsque ceux-ci ont intégré I'Union. Les PPM constituent un troisième groupe très hétérogène. Si les mieux placés (Algérie, Tunisie, Turquie) se rapprochent des PECO candidats exclus de la vague d'adhésion prévue en mai 2004 (Bulgarie, Roumanie), l'écart entre le Maroc et l'Égypte et les PECO les plus avancés est en revanche très élevé (de 1 à 4). Enfin, relativement aux pays d'Europe du Sud, l'écart est quasiment de 1 à 5 pour le Maroc et l'Égypte et de 2,5 à 3 pour le premier groupe (Algérie, Tunisie, Turquie). En outre, les taux de croissance du PIB sont très volatils, en particulier au Maroc en raison de sa dépendance aux fluctuations de la production agricole et dans les pays pétroliers du fait des variations des cours. Les taux de croissance moyens apparaissent relativement élevés (de 3 à $6 \%$ ) au cours des dernières périodes mais ils masquent des fluctuations importantes, dues à la succession de crises financières comme cela a été le cas en Turquie (TABleAu 1). 
Tableau 1 - Taux de croissance annuel moyen et PIB par habitant des pays méditerranéens, d'Europe du Sud et des candidats à l'adhésion

\begin{tabular}{lrrrrrrr}
\hline & \multicolumn{3}{c}{ Taux de croissance annuel } & \multicolumn{5}{c}{$\begin{array}{c}\text { PIB par habitant } \\
\text { (France }=1)\end{array}$} \\
& \multicolumn{3}{c}{ moyen, en \% } & \multicolumn{5}{c}{ \% } & \\
& $1992-$ & 1996 & 2000 & 1986 & 1993 & 1998 & 2003 \\
& 1995 & 1999 & 2003 & & & & \\
\hline Bulgarie & $-6,4$ & $-2,0$ & 4,8 & 0,37 & 0,26 & 0,22 & 0,26 \\
République Tchèque & 1,9 & 0,7 & 2,5 & n.d. & 0,59 & 0,61 & 0,63 \\
Estonie & $-7,4$ & 4,4 & 5,7 & n.d. & 0,30 & 0,36 & 0,42 \\
Hongrie & 0,1 & 3,7 & 3,8 & 0,58 & 0,45 & 0,49 & 0,56 \\
Lettonie & $-11,6$ & 4,9 & 6,6 & n.d. & 0,23 & 0,28 & 0,33 \\
Lituanie & $-11,5$ & 4,2 & 5,7 & n.d. & 0,30 & 0,32 & 0,34 \\
Pologne & 4,6 & 5,4 & 2,3 & 0,36 & 0,32 & 0,39 & 0,40 \\
Roumanie & 1,0 & $-2,1$ & 4,3 & 0,47 & 0,29 & 0,28 & 0,30 \\
Slovaquie & 0,2 & 4,2 & 3,5 & n.d. & 0,38 & 0,46 & 0,48 \\
Slovénie & 1,6 & 4,3 & 3,2 & n.d. & 0,60 & 0,69 & 0,74 \\
\hline UE-15 & 1,5 & 2,5 & 1,8 & & & & \\
France & 1,0 & 2,4 & 2,0 & 1 & 1 & 1 & 1 \\
Grèce & 0,8 & 3,2 & 4,1 & 0,68 & 0,65 & 0,67 & 0,70 \\
Portugal & 1,4 & 4,0 & 1,2 & 0,59 & 0,66 & 0,71 & 0,69 \\
Espagne & 1,3 & 3,7 & 2,8 & 0,71 & 0,77 & 0,81 & 0,84 \\
\hline Algérie & 0,6 & 3,3 & 3,7 & 0,31 & 0,23 & 0,22 & 0,21 \\
Maroc & $-0,5$ & 4,2 & 4,0 & 0,18 & 0,16 & 0,17 & 0,16 \\
Tunisie & 3,9 & 5,8 & 4,2 & 0,23 & 0,23 & 0,25 & 0,27 \\
Égypte & 1,8 & 5,4 & 3,3 & 0,15 & 0,15 & 0,15 & 0,15 \\
Turquie & 3,8 & 3,1 & 3,1 & 0,27 & 0,29 & 0,30 & 0,27 \\
\hline
\end{tabular}

Source: FMI, World Economic Outlook, calcul des auteurs.

\section{Les structures productives}

À ces différences de trajectoires de convergence réelle s'ajoutent des écarts importants dans les structures productives des économies. En 2000, la part de l'agriculture dans la valeur ajoutée (TABLEAU 2) est plus élevée dans les PPM (comprise entre 9 et $17 \%$ ), et dans les PECO les moins avancés (Bulgarie, Roumanie). Elle se situe entre 4 et $8 \%$ dans les nouveaux pays adhérents, comme dans les pays du Sud de l'Europe. Ce changement structurel est également palpable dans l'industrie dont la part dans la valeur ajoutée est comprise entre 21 et $30 \%$ dans les pays du Sud de l'Europe et s'élève à $30 \%$ dans les PECO (sauf en République Tchèque où le chiffre est proche de $40 \%$ ). On retrouve la même proportion dans les pays méditerranéens (à l'exception de l'Algérie). Dans les PECO, la contribution de I'industrie à la valeur ajoutée a diminué au profit des services. Ces derniers progressent également dans deux pays méditerranéens (Maroc, Tunisie) mais restent stables dans les autres pays de la zone. En Turquie, au Maroc et en Tunisie, la part des services dans la valeur ajoutée est relativement proche (de 55 à $60 \%$ ). L'Égypte et surtout l'Algérie restent à des niveaux inférieurs. 
Tableau 2 - $\quad$ Répartition sectorielle de la valeur ajoutée en 2000

En \%

\begin{tabular}{|c|c|c|c|c|c|c|c|c|c|}
\hline & \multicolumn{3}{|c|}{ Agriculture } & \multicolumn{3}{|c|}{ Industrie } & \multicolumn{3}{|c|}{ Services } \\
\hline & 1985 & 1995 & 2000 & 1985 & 1995 & 2000 & 1985 & 1995 & 2000 \\
\hline France & 3,8 & 3 & 3 & 28,3 & 24,4 & 25 & 61,1 & 65,3 & 72 \\
\hline Grèce & 14,7 & 9,1 & 4 & 27,2 & 20,7 & 30 & 48,9 & 62,6 & 66 \\
\hline Portugal & 7,1 & 4,7 & 4 & 37,4 & 27,4 & 30 & 54,1 & 59,1 & 66 \\
\hline Espagne & 5,6 & 4,3 & 8 & 27,2 & 21,3 & 21 & 58 & 63,7 & 71 \\
\hline Bulgarie & 11,9 & 12,9 & 14 & 62,5 & 33,9 & 30 & 25,6 & 53,2 & 56 \\
\hline République Tchèque & & 5 & 4 & 43 & 41 & & 53 & 55 & \\
\hline Estonie & 20,7 & 7,1 & 6 & 47 & 24,9 & 29 & 32,4 & 68 & 65 \\
\hline Hongrie & 17,9 & 7,1 & 6 & 47 & 34,6 & 34 & 35,2 & 58,3 & 61 \\
\hline Lettonie & & 9,8 & 4 & & 33,1 & 25 & & 57,1 & 70 \\
\hline Lituanie & 27,7 & 8,9 & 8 & 43,6 & 28,5 & 32 & 28,8 & 62,6 & 60 \\
\hline Pologne & 14,5 & 7,3 & 4 & 51 & 44 & 35 & 34,4 & 48,7 & 61 \\
\hline Roumanie & & 20,4 & 12 & & 32,7 & 34 & & 46,9 & 53 \\
\hline Slovaquie & 6,5 & 5,6 & 4 & 61,6 & 33,2 & 30 & 31,9 & 61,1 & 66 \\
\hline Slovénie & & 4,8 & 3 & & 38,5 & 38 & & 56,7 & 58 \\
\hline Algérie & 9 & 11,3 & 9 & 53,6 & 50,7 & 60 & 37,4 & 38 & 31 \\
\hline Égypte & 20 & 16,2 & 17 & 28,6 & 33,8 & 33 & 51,5 & 49,9 & 50 \\
\hline Maroc & 16,6 & 11,5 & 14 & 33,4 & 34 & 32 & 50 & 54,5 & 54 \\
\hline Tunisie & 17,3 & 13,5 & 12 & 34,1 & 32,6 & 29 & 48,6 & 53,9 & 59 \\
\hline Turquie & 20,4 & 15,9 & 15 & 21,2 & 24,9 & 25 & 58,4 & 59,2 & 59 \\
\hline
\end{tabular}

Source: Banque mondiale, World Development Indicators.

\section{Les différences de productivité en niveau}

Comme le montrent Chevallier et Ünal-Kesenci (2001), les écarts de productivité manufacturière observés par rapport à la France sont plus faibles que les écarts de revenus par tête (TABLEAU 3). Les niveaux de productivité des PPM ne se rapprochent pas du niveau français, à l'exception de la Turquie dont la productivité du travail progresse sensiblement plus vite que celle de la France pour la même période, malgré une forte croissance des effectifs employés.

Tableau 3 - $\quad$ Productivité par tête dans les secteurs manufacturiers

France $=100,1997$

\begin{tabular}{lccccc}
\hline & Espagne & Portugal & Turquie & Maroc & Égypte \\
\hline Total secteur manufacturier (1981) & 98 & 48 & 66 & $45(1988)$ & 20 \\
\hline Total secteur manufacturier (1997) & 92 & 43 & 74 & 34 & 21 \\
IAA & 92 & 37 & 62 & 50 & 21 \\
Textile & 98 & 50 & 86 & 46 & 18 \\
Chimie & 92 & 59 & 94 & 40 & 23 \\
Électrique-électronique & 83 & 39 & 79 & 39 & $27^{*}$ \\
\hline
\end{tabular}

* Électromécanique.

Source: Chevallier et Ünal-Kesenci (2001). 
On observe par ailleurs une très forte divergence des niveaux de productivité de l'industrie manufacturière de la plupart des PPM par rapport à l'Espagne (laquelle se rapproche étroitement de la France). En revanche, l'écart entre le niveau du Portugal et celui des PPM est faible. La Turquie présente même de meilleures performances que le Portugal dont le niveau de productivité recule de cinq points entre 1981 et 1997. Dans le secteur du textile-habillement, la productivité de la Turquie est proche de celle de l'Espagne et se situe à près de trente points au-dessus de celle du Portugal. Les autres PPM, I'Égypte en particulier, présentent néanmoins des niveaux très faibles dans tous les secteurs.

Enfin, plus généralement, pour les pays dont l'agriculture représente une part importante de l'activité, la faible productivité apparente de ce secteur par rapport au reste de l'économie pèse sur sa performance globale. Inversement, dans les pays développés, le recul de l'agriculture est plus sensible dans l'emploi que dans la valeur ajoutée, ce qui se traduit par une hausse de la productivité de l'ensemble de l'économie.

\section{Le capital humain et l'éducation}

Les niveaux de scolarisation diffèrent fortement au sein des pays de l'échantillon. Dans l'enseignement supérieur, le taux a fortement progressé partout (sauf en Lituanie). Initialement bas dans les PPM (près de $5 \%$ en 1980) à l'exception de l'Égypte (16\% en 1980), il double dans tous ces pays entre 1980 et 1995 (TABLEAU 4); sa progression est beaucoup plus forte en Turquie (quatre fois le niveau de 1980). La Turquie et l'Égypte présentent des taux dans l'enseignement supérieur proches de ceux de certains PECO (Roumanie, Slovaquie, République tchèque). L'écart avec les plus avancés des PECO et les pays d'Europe du Sud est très élevé. En Bulgarie, Estonie et Lituanie, ils se rapprochent de ceux des pays d'Europe du Sud et de la France (40\%). En outre, les taux dans l'enseignement secondaire et primaire sont beaucoup plus faibles pour les PPM que pour les PECO. Dans le secondaire, le taux n'excède pas $40 \%$ au Maroc. Toutefois, les niveaux de scolarisation doivent être interprétés avec prudence.

Au total, on constate une nette divergence structurelle entre les PPM et les pays de I'UE relativement à la position des PECO ou des pays d'Europe du Sud. Toutefois, on note un certain rattrapage pour la Turquie en termes de productivité. Les indicateurs retenus montrent que les PPM les plus avancés rejoignent à peine le niveau des PECO les moins avancés.

\section{LE CADRE INSTITUTIONNEL EURO-MÉDITERRANÉEN DE LIBÉRALISATION DES ÉCHANGES}

Dans la perspective d'une comparaison avec les conditions d'insertion des pays d'Europe Centrale et Orientale à I'UE, nous distinguons dans les PPM deux types de changements structurels et institutionnels, différents dans leur élaboration mais liés dans leurs implications: les conditions découlant directement de l'accord d'association euro-méditerranéen et celles externes à l'accord, se rapportant aux réformes structurelles dans les PPM. 
Tableau 4 - $\quad$ Taux de scolarisation brut par cycle d'éducation

En $\%$

\begin{tabular}{lrrrrrrrrr}
\hline & \multicolumn{4}{c}{ Primaire } & \multicolumn{3}{c}{ Secondaire } & \multicolumn{3}{c}{ Supérieur } \\
\hline & 1980 & 1990 & 1995 & 1980 & 1990 & 1995 & 1980 & 1990 & 1995 \\
\hline France & 111,10 & 108,50 & 106,10 & 84,60 & 98,50 & 111,30 & 25,30 & 39,60 & 51,00 \\
Grèce & 102,90 & 97,80 & 93,90 & 81,20 & 93,30 & 95,30 & 17,10 & 24,80 & 42,50 \\
Portugal & 123,20 & 123,40 & 128 & 37,20 & 67,40 & 106,3 & 10,70 & 23,20 & 37,20 \\
Espagne & 109,00 & 108,60 & 109,00 & 86,90 & 104,10 & 122,10 & 23,20 & 36,70 & 48,60 \\
Bulgarie & 97,80 & 97,60 & 96,80 & 84,50 & 75,20 & 78,00 & 16,20 & 31,10 & 39,40 \\
RépubliqueTchèque & 95,50 & 96,40 & 104,00 & 114,30 & 91,20 & 98,70 & 17,50 & 16,00 & 21,90 \\
Estonie & 103,00 & 110,70 & 91,30 & 126,60 & 101,90 & 103,70 & 24,50 & 26,00 & 38,10 \\
Hongrie & 96,40 & 94,50 & 103,20 & 69,50 & 78,60 & 97,80 & 14,10 & 14,00 & 23,80 \\
Lettonie & 102,00 & 94,20 & 88,70 & 99,40 & 92,70 & 85,00 & 23,50 & 25,00 & 25,70 \\
Lituanie & 78,80 & 90,70 & 95,90 & 113,60 & 91,90 & 84,20 & 34,80 & 33,80 & 28,20 \\
Pologne & 99,60 & 98,30 & 96,40 & 77,10 & 81,50 & 97,60 & 18,10 & 21,70 & 24,70 \\
Roumanie & 103,80 & 91,30 & 99,90 & 93,90 & 92,00 & 77,90 & 12,10 & 9,70 & 18,30 \\
Slovaquie &.. &.. & 102,80 &.. &.. & 93,70 & 18,30 & 18,60 & 20,20 \\
Slovénie & 98,20 & 108,30 & 97,70 & 38,10 & 91,10 & 90,50 & 20,20 & 23,70 & 33,30 \\
Algérie & 94,50 & 100,20 & 106,60 & 33,00 & 60,80 & 62,50 & 5,90 & 11,40 & 12,00 \\
Égypte & 73,10 & 93,80 & 99,80 & 50,50 & 76,20 & 76,50 & 16,10 & 15,90 & 20,30 \\
Maroc & 83,00 & 66,90 & 83,70 & 26,00 & 35,30 & 38,50 & 5,90 & 10,60 & 11,20 \\
Tunisie & 102,10 & 113,30 & 116,80 & 27,00 & 44,90 & 60,40 & 4,80 & 8,40 & 12,90 \\
Turquie & 96,00 & 99,00 & - & 35,00 & 47,00 & - & 5,40 & 13,10 & 18,20 \\
\hline
\end{tabular}

Source: Banque mondiale.

\section{Les conditions liées à l'accord lui-même}

Relativement à l'élargissement aux PECO, le partenariat Euromed présente une forte asymétrie au détriment des PPM. En raison du maintien des dispositions de la PAC et de la politique de fermeture des frontières pour les travailleurs, les barrières douanières sont maintenues pour les biens agricoles et les services. La libéralisation commerciale affecte davantage les partenaires méditerranéens, du Maghreb en particulier où les taux de protection sont parmi les plus élevés de la région. En revanche, sur les marchés européens, peu de barrières tarifaires à l'encontre des produits méditerranéens restent à démanteler.

Jusqu'à la signature du partenariat, les pays du Maghreb avaient accès aux marchés européens dans le cadre d'accords de coopération reconductibles périodiquement. Avec l'élargissement aux PECO et le démantèlement de l'accord multifibres, cet accès privilégié est remis en cause. Toutefois, I'UE peut accorder à ses partenaires de nouveaux avantages. Ainsi, les pays méditerranéens ont obtenu la possibilité de bénéficier de l'extension du "système paneuropéen de cumul de l'origine" : les marchandises ayant subi une transformation pourront accéder aux tarifs douaniers préférentiels en vigueur dans toute la zone. Cette extension devrait être favorable à l'augmentation des échanges euro-méditerranéens.

Par ailleurs, comparé aux accords de coopération renouvelés périodiquement, la pérennité du partenariat euro-méditerranéen pourrait permettre une plus grande crédibilité pour les inves- 
tissements directs étrangers ${ }^{4}$. Toutefois, les entraves au libre-échange sont plus souvent de nature non tarifaire ${ }^{5}$. Si certaines normes se justifient pour des raisons sanitaires, sociales, environnementales, etc., leur multiplication peut apparaître comme une protection déguisée ${ }^{6}$. La démarche de l'UE avec ses partenaires diffère selon qu'il s'agit des pays candidats à l'élargissement ou des PPM: les premiers, s'engageant à adopter tel quel "I'acquis communautaire", bénéficient pour cela d'une aide financière et technique substantielle, ce qui n'est pas le cas des seconds, pourtant fortement encouragés à harmoniser leurs normes avec celles de I'UE. De fait, la mise en conformité avec les normes communautaires, qui s'impose déjà aux PPM pour leurs exportations sur le marché européen, apparaît nécessaire pour attirer les investisseurs européens. Mais pour être attractifs à certains IDE, ces pays peuvent au contraire être tentés d'exploiter la possibilité de produire selon des normes moins exigeantes et de se spécialiser dans des industries polluantes ou dangereuses, au fur et à mesure que les normes se durcissent au Nord ${ }^{7}$.

Conséquence de ces asymétries, la levée des protections douanières provoque une aggravation des déficits commerciaux des PPM par rapport à I'UE à travers une augmentation mécanique des importations de produits européens. Le niveau élevé des tarifs douaniers entre les PPM eux-mêmes risque, en outre, de provoquer un effet de détournement de trafic au profit des produits européens. La concentration des échanges des PPM avec I'UE aggrave leur dépendance aux fluctuations de la croissance européenne. Seule une augmentation substantielle de leurs exportations peut réduire cette brutale augmentation des déficits commerciaux. Mais, les exportations des PPM ne peuvent augmenter que si les flux d'investissements directs étrangers affluent dans des secteurs tournés vers l'exportation.

Pourtant, la stagnation voire la dégradation des entrées d'IDE persiste, bien que les pays méditerranéens aient libéralisé leurs codes d'investissements et adopté des politiques favorables aux IDE. Leur faiblesse s'explique aussi par des facteurs institutionnels internationaux. Dans le cadre du partenariat Euromed, aucune institution similaire à la BERD n'a été mise en place ${ }^{8}$. Or celle-ci, par les garanties et incita-

\footnotetext{
4.Comme le montrent Cogneau, Dumont et Izzo (1998) et Mouhoud (1998), les investissements directs sont davantage influencés par le degré d'approfondissement de l'intégration régionale que par l'annonce d'un accord de libreéchange. Ceci est endogénéisé sous la forme de la réduction d'une prime de risque à l'investissement.

5. L'estimation du taux de protection moyen de l'ensemble de I'UE varie de $8 \%$ à $12,5 \%$ selon la méthode utilisée (CNUCED, OMC, CEPII pour la première; Messerlin, 2002 pour la seconde).

6. Millgram (2001) observe que l'utilisation parallèle d'autres instruments de politique commerciale (mesures antidumping et de sauvegarde, restrictions volontaires, contrôle des prix, accords préférentiels généraux ou spécifiques à certains produits, règles d'origine) a été accrue depuis que les derniers cycles de négociation du GATT et de I'OMC ont permis de larges réductions tarifaires (Millgram, 2001).

7. Ainsi, dans le cadre du vaste projet de réseau électrique euro-méditerranéen interconnecté "MedRing" ou "Anneau électrique euro-méditerranéen", auquel participent l'Algérie, l'Égypte, la Jordanie, le Liban, la Syrie, la Tunisie, la Turquie, I'Italie, la France, la Grèce et l'Espagne, il est prévu que des pays pétroliers et gaziers du Sud produisent de l'électricité pour les pays européens.

8. Une proposition minimale a finalement été adoptée par l'Europe en 2002 pour financer les investissements privés et d'infrastructure euro-méditerranéens avec le concours de la BEl.
} 
tions qu'elle avait apportées aux investisseurs, a été un puissant levier pour la promotion des IDE dans les PECO.

Toutefois, dans le cas des biens manufacturés, la progressivité du processus de démantèlement douanier atténue l'aggravation des déficits commerciaux. Par exemple, la Tunisie a même connu une hausse de la protection effective dans certains secteurs où le prix des inputs importés a baissé tandis que la protection du produit final était maintenue en raison de cette progressivité.

Au-delà du partenariat, les PPM peuvent simultanément abaisser certaines barrières douanières en faveur de leurs partenaires non européens. Cela favoriserait une ouverture multilatérale, dans l'esprit de l'OMC. Pour certains, cela permettrait de diversifier leurs échanges extérieurs et réduire la vulnérabilité de leurs économies aux fluctuations cycliques en Europe. Pour ceux dont les échanges sont plus diversifiés, cela minimiserait les risques de détournement de commerce (Talahite, 1996).

Ces effets négatifs ${ }^{9}$ à court terme de la libéralisation peuvent être contrebalancés par des effets dynamiques plus favorables liés au "choc" du libre-échange et aux changements structurels qu'il peut provoquer. L'un des principaux bénéfices du partenariat réside en effet dans I'incitation à l'accélération des réformes structurelles.

\section{Le cadre d'application de l'accord: la question des réformes structurelles}

En règle générale, les réformes structurelles sont engagées préalablement ou simultanément à l'intégration des économies dans un ensemble régional comme l'Union européenne. Dans les PPM, si les gouvernements ont réussi à engager des mesures de stabilisation macro-économiques, les réformes institutionnelles tardent à venir, en particulier dans le secteur fiscal et bancaire. Les organisations internationales considèrent ainsi les privatisations comme une réforme structurelle indispensable. Dans la plupart des PPM, leur mise en œuvre est lente ou bloquée. En Algérie et en Égypte, les marges de manœuvre dégagées par les ressources en hydrocarbures ont permis de retarder les décisions de réforme. En Tunisie, les zones franches de production ont constitué une alternative provisoire à la réforme de la législation et aux privatisations. Au Maroc, les privatisations du secteur bancaire et financier sont bien avancées.

Concernant le fonctionnement des marchés des biens, on peut distinguer deux types de PPM.

D’une part, les pays qui ont adopté dans les années 1960-1970 des politiques de développement dites "auto-centrées" et qui présentent un secteur productif largement étatisé. Leurs

9. Les simulations menées à l'aide de modèles EGC sur les pays de la région concluent que la signature d'un accord régional ne peut conduire qu'à un avantage statique limité et le plus souvent à une perte en bien-être, tandis que la libéralisation multilatérale du commerce de tous les biens sans discrimination entre les partenaires apporterait un gain supérieur. Voir pour la Tunisie, Rutherford et al. (1995), pour l'Égypte, Dessus et Suwa-Eisenmann (1998). 
secteurs industriels, liés à l'exploitation des ressources naturelles (pétrochimie, sidérurgie...) dépendent fortement des importations de biens intermédiaires et de biens d'équipement. Tournés vers le marché intérieur et à l'abri des barrières douanières, ils sont peu soumis aux normes du marché mondial. Dans la plupart des cas, l'industrie connaît des difficultés de gestion et souffre d'une très faible rentabilité. La majorité de ces pays, sous la pression du FMI, ont engagé des programmes de restructuration des entreprises publiques, mais ces réformes, qui nécessitent d'être étendues bien au-delà du secteur industriel (banques, institutions économiques, fiscalité, droits de propriété, marché du travail, lois sociales, etc.) avancent très lentement, en particulier les privatisations, condition posée par le FMI au rééchelonnement de la dette extérieure (Égypte en 1991, Algérie en 1994). Tant que ces réformes ne sont pas menées à terme, les potentialités réelles du secteur productif et ses capacités de reconversion sont difficiles à évaluer.

D'autre part, les pays comme le Maroc ou la Tunisie ont combiné politiques de substitution aux importations et reconversion du système productif vers les secteurs d'exportation. Ainsi, les exportations du Maroc se répartissent aujourd'hui entre les produits de consommation finale (agro-alimentaire, vêtements, cuir, électronique) et les produits intermédiaires (dérivés du phosphate). La Tunisie a opté pour les zones franches et la sous-traitance internationale essentiellement dans le textile.

Mais dans certains pays, les réformes structurelles se heurtent aux problèmes de transition fiscalo-douanière. Dans les systèmes dominés par la fiscalité indirecte - douanière en particulier - les pertes fiscales constituent l'effet négatif le plus évident de la libéralisation (estimées entre 9 et $35 \%$ du total des taxes perçues, soit 1 à $4 \%$ du revenu de chaque pays, Abed, 1998). Ceux qui devraient être les plus touchés sont le Liban et I'Algérie ${ }^{10}$ et, dans une moindre mesure, le Maroc et la Tunisie. S'y ajoutent les pertes de recettes sur les éventuels détournements du commerce avec des pays tiers, au profit de l'UE, ainsi que sur les taxes intérieures. Ces pays cherchent à substituer la fiscalité interne à la fiscalité externe dans un double objectif:

- trouver des ressources pour compenser la perte de recettes pour le budget de l'État (instauration ou perfectionnement de la TVA, amélioration de la collecte des impôts);

- réformer et rationaliser l'impôt afin de corriger les distorsions qu'il provoque et en faire un véritable instrument de politique économique.

Ces réformes ont été initiées dans certains PPM ${ }^{11}$ avant le partenariat Euromed (Abed, 1998). En outre, selon le FMI, I'efficience de I'administration fiscale pour la collecte de la TVA au Maroc et en Tunisie, est comparable à celle du Portugal. Toutefois, dans la majorité des PPM, le système fiscal demeure "complexe, inefficace et lourd à administrer".

10. Du fait de l'importance de ses importations de I'UE (56\% du total). Cependant, les ressources de l'État algérien proviennent en grande partie des revenus des hydrocarbures.

11. La TVA a été introduite au Maroc en 1986, en Tunisie en 1988, en Algérie en 1992 et la GST (generalized sales taxes) en 1991 en Égypte. 
La réforme du marché du travail est la plus difficile à mener. Le chômage de masse et les vagues de licenciements consécutives aux privatisations rendent très coûteuse la mise en place d'allocations pour les chômeurs. Dans le cadre du partenariat, I'UE propose des actions de formation de la main-d'œuvre mais ces pays connaissent un important chômage de diplômés. De plus, la mise aux normes européennes entraîne une déqualification de la maind'œuvre et une dévaluation des diplômes et savoirs faire locaux qui ne concerne pas seulement les métiers traditionnels, mais également les connaissances et l'expérience accumulée lors de la période de développement autocentré.

Enfin, la faible intégration entre les économies du Sud accentue l'effet centre-périphérie caractérisant l'accord d'association. Pourtant, les initiatives récentes visant à lever les obstacles aux échanges entre PEM (Protocole d'Agadir, Zone Arabe de Libre-Échange) pourraient mettre à profit les complémentarités évidentes entre les pays de la région.

Au total, aux difficultés liées à l'insuffisante couverture sectorielle et au caractère asymétrique du partenariat Euromed s'ajoutent les problèmes de faible efficacité des réformes économiques et sociales et de cloisonnement des pays du Sud de la Méditerranée entre eux. En dépit de ces difficultés structurelles et institutionnelles, il semble pertinent d'étudier la trajectoire de rattrapage des PPM par rapport aux pays de I'UE élargie en examinant particulièrement la transformation des structures de la spécialisation internationale et le rôle des IDE européens dans ces changements.

\section{LES INVESTISSEMENTS DIRECTS DANS LES PAYS MÉDITERRANÉENS ET L'ÉVOLUTION DE LEUR SPÉCIALISATION INTERNATIONALE}

Les échanges internes à l'Union européenne représentent plus des deux tiers du commerce des pays membres (TABLEAU 5). Dès lors, la part des pays méditerranéens et des PECO est négligeable dans le commerce de l'UE. En revanche, la part de l'UE dans les exportations de ces deux zones est très importante, tandis que les échanges commerciaux entre les PPM euxmêmes sont négligeables. L'Espagne et surtout le Portugal ont, depuis 1970, largement redirigé leurs exportations vers les membres de la Communauté européenne. Dans le cas des PECO, près de $68 \%$ des exportations sont dirigées vers I'UE. De même, le Maroc, et plus encore la Tunisie, privilégient I'UE comme destination de leurs exportations (près de $81 \%$ dans le dernier cas). À l'inverse, la Grèce, l'Égypte et la Turquie ont une structure commerciale moins orientée vers l'Europe.

\section{La spécialisation des pays périphériques et méditerranéens}

La littérature montre globalement que l'UE présente une spécialisation internationale faible et disparate. Les pays du noyau dur sont les plus diversifiés tandis que les États membres situés à la périphérie, en particulier le Portugal et la Grèce, apparaissent plus spécialisés (TABLEAU 6). En évolution, la mise en place du Marché unique implique généralement un 
accroissement des disparités dans les structures industrielles mais les pays sont affectés différemment selon qu'ils se situent au centre ou à la périphérie. Pour ceux du noyau dur, l'indice de spécialisation croît plutôt en fin de période tandis que les autres connaissent un processus de déspécialisation en début de période (Amiti, 1999; Midelfart-Knarvik et al., 2000).

Tableau 5 - $\quad$ Poids de I'UE dans les exportations de I'UE-15, des PECO et des pays méditerranéens

\begin{tabular}{|c|c|c|c|c|c|}
\hline & 1970 & 1980 & 1990 & 1995 & 1999 \\
\hline UE-15 & 59,92 & 60,78 & 66,56 & 61,64 & 61,31 \\
\hline Espagne & 51,69 & 53,96 & 71,45 & 72,18 & 71,16 \\
\hline Grèce & 56,13 & 49,53 & 68,36 & 59,97 & 50,02 \\
\hline Portugal & 52,40 & 66,02 & 80,42 & 79,92 & 82,20 \\
\hline PECO-10 & - & - & - & 60,19 & 67,29 \\
\hline Maroc & 75,99 & 68,99 & 71,52 & 71,76 & 66,56 \\
\hline Tunisie & 64,81 & 84,22 & 77,67 & 79,60 & 81,19 \\
\hline Algérie & 81,27 & 42,91 & 67,86 & 67,27 & 61,26 \\
\hline Égypte & 25,46 & 65,84 & 59,05 & 55,04 & 47,09 \\
\hline Turquie & 53,64 & 47,69 & 55,87 & 50,86 & 54,46 \\
\hline
\end{tabular}

Source: CEPII, Bases de données CHELEM, calculs des auteurs.

Tableau 6 - $\quad$ Indice de Krugman (spécialisation relative vs UE-15)

\begin{tabular}{lcccc}
\hline & $87-89$ & $90-92$ & $93-95$ & $96-99$ \\
\hline Espagne & 0,509 & 0,515 & 0,521 & 0,524 \\
Portugal & 0,952 & 0,922 & 0,913 & 0,831 \\
Grèce & 1,189 & 1,151 & 1,066 & 1,024 \\
\hline Pologne & & & 0,832 & 0,745 \\
Hongrie & & 0,622 & 0,655 \\
Slovénie & & 0,662 & 0,669 \\
Slovaquie & & 0,681 & 0,661 \\
Estonie & & & 0,949 & 0,977 \\
République Tchèque & & & 0,586 & 0,563 \\
Lettonie & & & 0,990 & 1,007 \\
Lituanie & & & 0,889 & 0,945 \\
\hline Bulgarie & & 0,980 & 1,086 \\
Roumanie & & 1,862 & 1,862 & 1,826 \\
\hline Algérie & 1,832 & 1,398 & 1,396 & 1,370 \\
Égypte & 1,527 & 1,454 & 1,436 & 1,448 \\
Maroc & 1,446 & 1,305 & 1,312 & 1,375 \\
Tunisie & 1,344 & 1,171 & 1,126 & 1,043 \\
Turquie & 1,096 & & & \\
\hline
\end{tabular}

Source: CEPII, Bases de données CHELEM, calculs des auteurs. 
La méthode d'évaluation de l'intensité de la spécialisation internationale appliquée aux pays d'Europe du Sud, aux nouveaux pays membres et aux pays méditerranéens se fonde sur des indices de Krugman calculés sur données d'exportations vers I'UE-15 avec une désagrégation relativement fine à 72 produits (source: CEPII, bases de données CHELEM). L'indice appliqué se définit ainsi :

$$
\begin{aligned}
& K_{i}(t)=\sum_{k} \operatorname{abs}\left(v_{i}^{k}(t)-\bar{v}_{i}^{k}(t)\right) \operatorname{avec} v_{i}^{k}(t) \equiv x_{i}^{k}(t) / \sum_{k} x_{i}^{k}(t) \\
& \text { et } \bar{v}_{i}^{k}(t) \equiv \sum_{j \neq i} x_{i}^{k}(t) / \sum_{k} \sum_{j \neq i} x_{i}^{k}(t)
\end{aligned}
$$

II renseigne sur l'écart entre la structure de production $v_{i}^{k}(t)$ du pays $i$ et $\bar{v}_{i}^{k}(t)$ la moyenne des structures de l'ensemble des pays de I'UE-15, avec $k$ les différents secteurs de l'économie. II prend des valeurs comprises entre 0 pour une spécialisation nulle et 2 pour une spécialisation complète (Krugman, 1991). Parmi les États-membres d'Europe du Sud, I'Espagne est la moins spécialisée; l'indice apparaît en outre relativement stable. Le Portugal et la Grèce, initialement plus spécialisés, connaissent un processus de diversification des activités, ce qui reflète un rapprochement de leurs structures industrielles avec la moyenne des Étatsmembres.

La majorité des PECO, en particulier les plus avancés d'entre eux, sont plus diversifiés que le Portugal et la Grèce. La République Tchèque par exemple affiche un niveau de spécialisation voisin de celui de l'Espagne. La Bulgarie et la Roumanie dont la date d'adhésion est reportée à 2007 et les États Baltes, moins avancés et plus éloignés géographiquement de l'Union, présentent les indices les plus élevés. La spécialisation de la Lettonie est la plus élevée.

Les nouveaux pays membres sont généralement spécialisés dans les secteurs intensifs en ressources naturelles (métallurgie, bois) et en travail (textile-habillement) tandis qu'ils restent désavantagés dans les secteurs à forte intensité capitalistique comme les machines, les biens d'équipement ou encore les produits chimiques (Freudenberg et Lemoine, 1999). Toutefois, des divergences nettes apparaissent et le contenu de la spécialisation se modifie pour plusieurs d'entre eux. Les plus avancés disposent désormais d'avantages dans certains secteurs de pointe ou à forte intensité capitalistique tout en conservant des avantages non négligeables là où la valeur ajoutée est plus faible (Boillot, 2003). La Hongrie par exemple, possède des avantages comparatifs dans l'électronique ou l'informatique, secteurs dans lesquels les autres PECO sont plutôt désavantagés. L'Estonie présente désormais un avantage dans le matériel de télécommunications tandis que les autres pays baltes et la Bulgarie ou la Roumanie restent marqués par une spécialisation forte dans les secteurs traditionnels (produits agricoles, métallurgie, textile). La Pologne également exporte pour l'essentiel des biens issus de ces secteurs (charbon, textile, métallurgie).

Les pays méditerranéens sont les plus spécialisés de l'échantillon, à l'exception de la Turquie dont l'indice, qui décroît rapidement, est proche de ceux des pays candidats les moins avancés. Le Maroc, la Tunisie et l'Égypte présentent des spécialisations relativement proches et 
très marquées à l'égard de l'UE, en raison du poids que représente un petit nombre d'activités pour lesquelles ils ont des avantages comparatifs. C'est par exemple le cas du textile pour le Maroc et la Tunisie. Ils possèdent également des avantages comparatifs dans des secteurs agricoles et/ou intensifs en ressources naturelles. Ces pays exportent une part non négligeable de biens manufacturés, mais à faible valeur ajoutée. La très forte spécialisation de l'Algérie est due à ses exportations de pétrole et de gaz. Enfin, l'Égypte possède également des avantages très marqués dans les produits pétroliers (pétrole et produits raffinés).

Le TABLEAU 7 renseigne sur la similarité des structures d'exportation entre les différents pays

de l'échantillon mesurée par l'indice de Finger-Kreinin (1979). II se définit de la manière suivante: $\sum_{i} \min \left[\frac{X_{i j k}}{X_{i}^{*}} ; \frac{X_{l j k}}{X_{l}^{*}}\right]$ où $X_{i j k}$ et $X_{l j k}$ désignent les exportations des pays $i$ et $/$ vers le pays $j$ en produit $k_{i} X_{i}^{*}$ et $X_{l}^{*}$ désignent les exportations totales des pays $i$ et I.

Plus les indices sont élevés, plus les structures commerciales des pays sont proches. Pour I'Espagne et les PECO les plus avancés, les indices proches de 0,75 révèlent une structure des échanges assez convergente par rapport à l'UE. On retrouve globalement la même hiérarchie que pour l'indicateur de PIB par tête et les indices de spécialisation. En revanche, le Portugal et la Grèce avoisinent les PECO du deuxième groupe et la Turquie. À nouveau, les deux groupes de PECO divergent nettement entre eux tandis que les PPM affichent une similarité de leurs échanges avec l'UE-15 presque deux fois plus faible que pour les PECO les moins avancés. De plus, l'indice permet de repérer les éventuels effets de concurrence ou de complémentarité entre pays périphériques. De ce point de vue, les PECO semblent davantage en concurrence entre eux et avec l'Europe du Sud et la Turquie qu'avec les pays méditerranéens. Parmi les PPM, la Turquie est la plus proche des PECO et des pays d'Europe du Sud. Le Maroc et la Tunisie sont similaires entre eux et vis-à-vis des PECO les moins avancés. L'Algérie est le pays le plus éloigné.

\section{Le rôle des IDE dans le processus de rattrapage et de transformation de la spécialisation internationale: éléments empiriques}

Dans le cas de l'Espagne et de certains PECO, les IDE contribuent à l'évolution de la spécialisation vers un scénario de type intra-branche. Les investissements directs s'effectuent généralement par le biais d'opérations de privatisation des grandes sociétés existantes ou d'accords de coopération et de joint-venture avec des firmes locales. Tout en partageant les risques liés à la rentabilité future des investissements, les firmes étrangères se trouvent en bonne position pour conquérir les marchés locaux. Elles apportent leurs savoir-faire et leurs structures de management, ce qui contribue à faciliter la transition vers des structures modernes. D'emblée, les PECO se sont insérés dans les nouvelles formes d'IDE qui, en principe, facilitent les transferts de technologie et de savoir-faire. 
Tableau 7 - $\quad$ Indice de similarité de Finger-Kreinin

\begin{tabular}{|c|c|c|c|c|c|c|c|c|c|c|}
\hline 1999 & $\begin{array}{c}\text { UE- } \\
15\end{array}$ & Espagne & Portugal & Grèce & $\begin{array}{c}\text { PECO- } \\
10\end{array}$ & $\begin{array}{c}\text { PECO } \\
\text { groupe } \\
1\end{array}$ & $\begin{array}{c}\text { PECO } \\
\text { groupe } \\
2\end{array}$ & Maroc & Tunisie & Algérie Égypte \\
\hline \multicolumn{11}{|l|}{ UE-15 } \\
\hline Espagne & 0,739 & & & & & & & & & \\
\hline Portugal & 0,594 & 0,637 & & & & & & & & \\
\hline Grèce & 0,485 & 0,553 & 0,482 & & & & & & & \\
\hline $\begin{array}{l}\text { PECO-10 } \\
\text { PECO }\end{array}$ & 0,730 & 0,704 & 0,687 & 0,514 & & & & & & \\
\hline $\begin{array}{l}\text { groupe } 1 \\
\text { PECO }\end{array}$ & 0,753 & 0,714 & 0,682 & 0,463 & & & & & & \\
\hline groupe 2 & 0,499 & 0,509 & 0,554 & 0,622 & & 0,578 & & & & \\
\hline Maroc & 0,273 & 0,335 & 0,376 & 0,467 & 0,341 & 0,294 & 0,481 & & & \\
\hline Tunisie & 0,305 & 0,302 & 0,453 & 0,453 & 0,397 & 0,364 & 0,507 & 0,611 & & \\
\hline Algérie & 0,081 & 0,064 & 0,045 & 0,144 & 0,068 & 0,052 & 0,134 & 0,057 & 0,088 & \\
\hline Égypte & 0,333 & 0,320 & 0,384 & 0,534 & 0,393 & 0,335 & 0,521 & 0,342 & 0,359 & 0,471 \\
\hline Turquie & 0,502 & 0,562 & 0,594 & 0,608 & 0,576 & 0,548 & 0,576 & 0,488 & 0,454 & $0,471 \quad 0,462$ \\
\hline
\end{tabular}

Source: CEPII, Bases de données CHELEM, calculs des auteurs.

Après 1989, les IDE se sont massivement dirigés vers l'Europe de l'Est. Potentiellement, ils pèsent donc sur la structure des exportations et la dynamique de la spécialisation internationale. Rapportés aux flux dirigés vers les pays en développement, la part des pays candidats n'a cessé de s'accroître au cours des années 89-2002 passant de $5 \%$ environ au début des années quatre-vingt-dix à près de $10 \%$ au tournant du siècle (TABLEAU 8). Provenant essentiellement de I'Union européenne, les IDE à l'Est se concentrent toutefois dans trois pays: Pologne, République Tchèque et Hongrie. À eux trois, ils regroupent plus des trois quarts des entrées d'investissements en Europe Centrale et Orientale.

Tableau 8 - $\quad$ Flux annuels moyens d'investissements directs

En millions de dollars

\begin{tabular}{lrrr}
\hline & $1989-1994$ & $1995-1998$ & $1999-2002$ \\
\hline Monde & 201398 & 471678 & 986764 \\
Pays développés & 139314 & 291915 & 748721 \\
Pays en développement & 58448 & 162288 & 211732 \\
\hline Union européenne & 78958 & 150748 & 480812 \\
Europe du Sud & 14034 & 10804 & 30992 \\
PECO-10 & 2584 & 12777 & 20618 \\
PPM-5 & 2282 & 2868 & 5055 \\
\hline Algérie & 27 & 258 & 802 \\
Égypte & 839 & 799 & 864 \\
Maroc & 353 & 565 & 1259 \\
Tunisie & 355 & 409 & 614 \\
Turquie & 708 & 838 & 1517 \\
\hline PPM-5/PVD (en \%) & 3,90 & 1,77 & 2,39 \\
PECO-10/PVD (en \%) & 4,42 & 7,87 & 9,74 \\
\hline
\end{tabular}

Source: CNUCED, calcul des auteurs. 
Le diagnostic est moins favorable ${ }^{12}$ pour les PPM. Malgré la mise en œuvre de réformes et les initiatives d'intégration régionale, ils restent dans l'ensemble peu attractifs aux entrées d'investissements directs. La part qu'ils représentent dans les pays en développement a baissé entre 1989 et 2002. Les montants, bien qu'en augmentation depuis 2001, en particulier au Maroc et en Turquie, sont restés relativement modestes dans tous les pays de la région.

Plus que leur montant, c'est la répartition sectorielle des IDE qui importe pour l'évaluation de la transformation de la spécialisation internationale des pays d'accueil. Les données restent toutefois rares pour les PECO et très marginales pour les PPM. Parmi les PECO, une enquête d'Eurostat (2002) distingue trois groupes.

Le premier est constitué uniquement de la Bulgarie, seul pays où les stocks d'IDE sont dominés par l'industrie manufacturière. L'agroalimentaire, la métallurgie et la chimie sont les principaux secteurs qui attirent des IDE.

Un second groupe est composé de la Pologne, la Slovénie, la République Tchèque et la Slovaquie où les services représentent 51 à $53 \%$ du stock total. Le commerce et les activités financières captent l'essentiel des flux entrants. Dans l'industrie, des différences importantes existent entre les pays: I'industrie alimentaire occupe une place importante en Pologne, c'est le cas de la métallurgie et de la fabrication de machines et équipements en Slovaquie et de chimie en Slovénie.

Enfin, un dernier groupe est constitué des États Baltes où la part des services, supérieure aux deux tiers du stock total dépasse largement la moyenne des autres pays. Le commerce, les activités financières et les transports et télécommunications sont les principaux secteurs investis par les entreprises étrangères.

Contrairement aux PECO, les PPM reçoivent plutôt des IDE dans les secteurs manufacturiers intensifs en main-d'œuvre et dans les secteurs primaires. Les informations disponibles montrent une forte concentration des IDE sur un petit nombre de secteurs (El Hédi Lahouel, 2000). En Tunisie, l'énergie, en particulier le secteur gazier, attire environ deux tiers des entrées d'IDE, le reste se répartissant entre le tourisme et les industries légères à faible intensité en compétences et bas coût de main-d'œuvre. Au Maroc, ils privilégient les services - plus précisément la finance et le tourisme - tandis qu'en Égypte et en Algérie, c'est majoritairement vers le secteur pétrolier qu'ils se dirigent.

En termes de déterminants, plusieurs facteurs expliquent la montée en puissance du stock d'IDE dans les PECO. En premier lieu, le nouvel environnement institutionnel a permis aux investisseurs de réaliser des opérations jusqu'alors impossibles. La transition et la perspective affirmée de l'intégration ont ensuite concouru à renforcer leur attractivité. En second lieu, la proximité géographique, la disponibilité de main-d'œuvre relativement bon marché et qualifiée et un marché potentiel important constituent des avantages décisifs (Dupuch et Milan,

12. Les données d'IDE dans la zone méditerranée sont à prendre avec prudence. Par exemple, les données fournies par la CNUCED tendent à surévaluer les flux en direction de cette région, si on les compare aux chiffres de balance des paiements des pays investisseurs (Alessandrini et Resmini, 2000). 
2003). En outre, les processus de privatisation sont intervenus alors que la concurrence était encore peu intense, ce qui a permis aux firmes étrangères de bénéficier d'avantages du type premier entrant.

Les PPM disposent en principe de certains avantages permettant d'attirer des investisseurs étrangers: le dispositif de barrières douanières peut attirer des investissements de contournement, les FMN s'implantant dans le pays pour vendre sur le marché intérieur en étant plus compétitives que si elles exportaient depuis leur pays d'origine. Au cours des deux dernières décennies, ce type d'investissement a bénéficié par exemple à l'Algérie pour l'énergie et l'extraction des matières premières ou dans l'industrie en Égypte ou en Turquie où les implantations étrangères étaient constituées d'unités d'assemblage visant à fournir les marchés locaux (Alessandrini et Resmini, 2000). II s'agit en fait d'un avantage fragile: le maintien de barrières tarifaires entre les pays de la zone révèle l'absence de perspective d'intégration Sud-Sud et de réalisation d'un vaste marché méditerranéen. L'asymétrie des protections tarifaires peut également fonder la stratégie d'implantation de firmes non européennes utilisant les PPM comme base arrière pour fournir le marché européen. Toutefois, cet avantage n'est aujourd'hui que transitoire, étant donné les perspectives de démantèlement des tarifs douaniers. La faiblesse des coûts salariaux dans des secteurs intensifs en main-d'œuvre constitue un avantage plus tangible. Malgré une faible productivité apparente, les investissements semblent se concentrer dans des secteurs plutôt traditionnels comme le textile ou l'agroalimentaire. De plus, la faiblesse de la taille du marché joue de manière défavorable.

De même, l'environnement local affecte l'attractivité de la région. Les politiques de restrictions aux participations étrangères suivies par plusieurs pays, l'instabilité macro-économique, la faiblesse du potentiel de croissance contribuent à expliquer que les accords de libéralisation, contrairement aux attentes, n'aient pas eu pour conséquence l'intensification des entrées d'IDE dans la région (El Hédi Lahouel, 2000).

L'effet des investissements directs sur la spécialisation n'est pas neutre, en particulier dans les pays candidats où le poids des investisseurs étrangers est désormais prépondérant. Ainsi, l'émergence de nouvelles spécialisations en Europe centrale doit beaucoup à I'IDE et aux importants transferts technologiques opérés ces dernières années. Certains pays, en attirant des firmes étrangères dans des secteurs à rendements croissants seraient susceptibles de connaître un scénario de rattrapage rapide. Par contre, les PECO les moins avancés resteraient enfermés dans des spécialisations plus traditionnelles. Parmi les pays plus avancés, les IDE contribuent à la transformation des spécialisations sectorielles et à leur montée en contenu en R.\&D. et en travail qualifié. Malgré la faiblesse relative des dépenses de R.\&D., certains PECO qui disposaient déjà d'un système national d'innovation sont en train de passer d'une spécialisation fondée sur la basse technologie à des secteurs de moyenne voire de haute technologie. En 1996, les PECO se positionnent essentiellement sur des produits de bas et de milieu de gamme mais des différences fortes caractérisent la structure de leurs échanges: $44 \%$ des exportations hongroises et $73 \%$ des exportations roumaines sont 
constituées de biens de bas de gamme tandis que la part des biens de haut de gamme varie de $31 \%$ en Slovénie et $28 \%$ en Hongrie à $8 \%$ en Roumanie. En évolution, seuls les pays d'Europe Centrale voient progresser les exportations de biens de haut de gamme, ce qui s'oppose à l'hypothèse d'un enfermement dans les spécialisations actuelles (Freudenberg et Lemoine, 1999).

Toutefois, les transferts de technologie sont conditionnés par la capacité d'absorption des PECO. Une enquête sur les IDE en République Tchèque (Kinoshita, 2000) montre que l'effet de la R.\&D. sur la croissance de la productivité n'est pas lié aux capacités d'innovation mais repose indirectement sur la capacité d'absorption. L'importance des spillovers consécutifs aux IDE varie selon les secteurs. Ils sont particulièrement significatifs dans les machines électriques et l'électronique. À l'opposé, aucun effet tangible n'apparaît dans les secteurs agroalimentaire et minéraux non métalliques. Dans les PPM, les retombées de la présence de firmes étrangères en matière de transfert de technologie, de connaissance et de savoir-faire restent marginales. Le risque d'un verrouillage à moyen terme des structures productives des PPM dans des spécialisations traditionnelles se confirme.

\section{Qui emprunte la voie de la spécialisation intra-branche?}

Les indices de spécialisation corroborent largement l'évolution du commerce intra-branche des pays du Sud de l'Europe, des PECO et des pays méditerranéens. Les échanges européens sont constitués majoritairement de flux de type intra-branche, en particulier en différenciation verticale dont la progression est sensible après 1985. Les pays périphériques restent toutefois en marge de cette tendance où le commerce inter-branche domine toujours malgré un rattrapage tangible de la part de l'Espagne et dans une moindre mesure du Portugal. L'analyse de la nature du commerce UE-PECO en 1996 que les échanges inter-branche dominent entre I'UE-12 et les PECO dans leur ensemble, avec des différences très marquées entre pays (TABleau 9).

Les pays d'Europe centrale (République Tchèque, Hongrie et Slovénie) enregistrent la part de commerce intra-branche la plus élevée, dépassant le Portugal et la Grèce. La Pologne et la Slovaquie occupent une position intermédiaire tandis que les échanges inter-branche dominent largement dans les Balkans et les pays baltes (entre 85 et $95 \%$ ). Parmi les PPM, la Tunisie et la Turquie sont proches des PECO les moins avancés avec près de $20 \%$ d'échanges intra-branche. Le commerce de biens différenciés verticalement domine également, ce qui signifie que les produits importés et exportés depuis I'UE diffèrent largement par la qualité. À I'inverse, l'Algérie, l'Égypte et le Maroc conservent une structure d'échanges presque exclusivement inter-branche. Au niveau des secteurs, les échanges intra-branche sont nettement plus significatifs dans les nouveaux secteurs d'exportation (matériel électrique et électronique) que dans les activités plus traditionnelles (20\% pour l'électronique en Égypte, environ $25 \%$ des échanges de pièces mécaniques entre I'UE et le Maroc, plus de $45 \%$ pour le matériel électrique et $20 \%$ pour le matériel électronique). Entre I'UE et la Turquie, $26 \%$ des échanges sont intra-branche dans la mécanique et $29 \%$ pour les véhicules. La Tunisie est le 
pays dans lequel les textiles représentent la plus forte proportion des échanges intra-branche. Néanmoins, ces caractéristiques tiennent davantage à la nature de ces secteurs, plus intensifs en biens intermédiaires qu'aux capacités des pays à attirer des IDE et à développer des avantages comparatifs dans ces secteurs.

Tableau 9 - $\quad$ Part des échanges intra-branche dans les échanges avec l'UE

\begin{tabular}{|c|c|c|c|}
\hline \multirow{2}{*}{1996} & \multicolumn{2}{|c|}{ Intra branche en différenciation } & \multirow{2}{*}{ Inter-branche } \\
\hline & Horizontale & Verticale & \\
\hline République Tchèque & 9,6 & 38,1 & 52,3 \\
\hline Hongrie & 6,4 & 30,9 & 62,7 \\
\hline Slovénie & 4,8 & 27,3 & 67,9 \\
\hline Slovaquie & 5,7 & 19,7 & 74,6 \\
\hline Pologne & 2,7 & 20,6 & 76,6 \\
\hline Roumanie & 1,7 & 13,3 & 85 \\
\hline Bulgarie & 2,6 & 11,2 & 86,3 \\
\hline Lituanie & 1,1 & 5,9 & 92,9 \\
\hline Lettonie & 0,9 & 4 & 95,1 \\
\hline Estonie & 0,8 & 3,5 & 95,7 \\
\hline Algérie & 0,6 & 1,1 & 98,4 \\
\hline Égypte & 1,0 & 3,1 & 95,9 \\
\hline Maroc & 3,4 & 5,4 & 91,2 \\
\hline Tunisie & 4,1 & 15,2 & 80,6 \\
\hline Turquie & 6,1 & 10,2 & 83,7 \\
\hline UE-12 (1980) & 18,08 & 35,36 & 46,57 \\
\hline UE-12 (1985) & 17,94 & 34,54 & 47,52 \\
\hline UE-12 (1994) & 19,23 & 42,28 & 38,5 \\
\hline Grèce (1980) & 2,02 & 11,33 & 86,65 \\
\hline Grèce (1996) & 2,80 & 9,60 & 87,60 \\
\hline Espagne (1985) & 10,12 & 26,35 & 63,52 \\
\hline Espagne (1996) & 18,50 & 37,10 & 44,50 \\
\hline Portugal (1985) & 4,13 & 10,45 & 85,42 \\
\hline Portugal (1996) & 11,1 & 24,80 & 64,10 \\
\hline
\end{tabular}

Sources: Freudenberg et Lemoine, 1999; Fontagné, Freudenberg et Péridy, 1998.

La structure des échanges commerciaux suggère pour certains pays une tendance à la diversification, en particulier pour un petit groupe de PECO qui possède désormais des avantages comparatifs dans des secteurs à plus forte valeur ajoutée. Les pays méditerranéens, pour leur part, cantonnés dans des secteurs à faible valeur ajoutée, intensifs en main-d'œuvre ou en ressources, apparaissent très complémentaires aux pays membres de I'UE. Certaines études montrent toutefois des éléments de diversification des échanges commerciaux et d'amélioration de la qualité (Chevallier et Freudenberg, 2001). Mais cela reste minoritaire, tant les secteurs à forte intensité capitalistique demeurent marginaux dans les exportations des pays méditerranéens. Par ailleurs, le fait que la Tunisie développe une forte proportion de ses 
échanges intra-branche dans le secteur textile ne peut constituer un avantage tangible tant ce secteur est menacé par l'ouverture du marché européen après le démantèlement de l'accord multifibres.

\section{Conclusion}

Des différences structurelles importantes séparent les PPM des pays de I'UE relativement à la position des PECO ou des pays d'Europe du Sud. Les PECO semblent pour l'ensemble des indicateurs retenus nettement mieux placés que les PPM. Ces derniers présentent une faible convergence réelle et structurelle, même si l'observation de certaines variables révèle un véritable rattrapage de certains pays du Sud de la Méditerranée tels que la Turquie en termes de productivité. À l'inverse, certains pays d'Europe du Sud et de l'Est connaissent une dégradation de leur position structurelle (Bulgarie, Roumanie, Portugal).

En outre, la faible attractivité des PPM aux IDE indique que les conditions d'accès au marché européen se sont probablement dégradées malgré la signature d'accords d'association. La levée des barrières protectionnistes ne suffit pas à réduire les réticences des investisseurs étrangers liées à la faiblesse des réformes mises en œuvre à l'échelle nationale ou à l'inadéquation des infrastructures. Si le rôle des privatisations dans l'accélération de I'IDE doit être souligné, de même que le caractère durable de l'accord autorisant à l'avenir une diversification des IDE en particulier dans des secteurs à plus forte intensité capitalistique, de nombreux obstacles continuent de contrarier l'attractivité des PPM, en particulier un marché local très restreint que l'accord ne modifie pas. En raison du maintien de barrières tarifaires au sein des pays partenaires, les marchés restent cloisonnés, ce qui limite les possibilités d'expansion du commerce intra-régional.

Les pays méditerranéens apparaissent très spécialisés et leur industrie reste cantonnée dans des secteurs à faible valeur ajoutée, intensifs en main-d'œuvre ou en ressources et apparaissent de ce fait très complémentaires aux pays membres de l'UE. Si certaines études montrent des éléments de diversification des échanges commerciaux et d'amélioration de la qualité, cela reste très minoritaire. Par ailleurs, le développement d'avantages comparatifs dans des secteurs comme le textile ne peut constituer un avantage tangible à long terme en raison de I'intensification de la concurrence de la Chine consécutive à son adhésion à l'OMC et les perspectives de démantèlement de l'accord multifibres.

Au total, la physionomie de la géographie européenne ne ressemble plus à la vision optimiste selon laquelle les États membres convergent entre eux et développent des échanges de biens différenciés peu enclins à subir des chocs de nature asymétrique. Les différences entre les zones géographiques qui composent I'Union et son voisinage sont de plus en plus prononcées. La structure des échanges commerciaux suggère pour certains pays une tendance à la diversification, en particulier pour un petit groupe de PECO qui possède désormais des avantages comparatifs dans des secteurs à plus forte valeur ajoutée. Les autres PECO et certains 
pays d'Europe du Sud subissent également des différenciations fortes à l'égard du cœur de I'Union européenne. Le troisième groupe composé des voisins méditerranéens est également hétérogène puisque la Turquie se distingue par une amorce de convergence structurelle ${ }^{13}$.

S. D., E.M. M. \& F. T.

\section{RÉFÉRENCES}

Abed, G.T., 1998. Trade liberation and tax reform in the Southern Mediterranean region, IMF Working Paper 98/49.

Alessandrini, S., Resmini, L., 2000. FDI in the Mediterranean region: A comparison with CEE experience, préparé dans le cadre du programme de recherche FEMISE, janvier.

Amiti, M., 1999. Specialization patterns in Europe, Weltwirtschaftliches Archiv 135 (4), 573-593.

Boillot, J.J., 2003. L'Élargissement de I'UE: un défi économique pour tous, La Documentation Française.

Chevallier, A., Freudenberg, M., 2001. La nature des échanges euro-méditerranéens et les perspectives d'intégration régionale, dans Dessus, S., Devlin, J., Vers une intégration régionale arabe et euro-méditerranéenne, Paris, Centre de développement de I'OCDE, 63-91.

Chevallier, A., Lemoine, F., Nayman, L., 1999. "L’Union européenne et sa périphérie: conséquences de l'intégration commerciale de l'Europe centrale", Revue Économique 50 (6), novembre.

Chevallier, A., Ünal-Kesenci, D., 2001. La productivité des industries méditerranéennes, CEPll, Document de travail 16-2001, décembre.

Cogneau, D., Dumont, J.C., Izzo, C., 1998. Intégration régionale, investissements directs et migrations dans l'espace euroméditerranéen, dans Migrations, libre-échange et intégration régionale dans le Bassin méditerranéen, OCDE.

Dessus, S., Suwa-Eisenmann, A., 1998. Trade integration with Europe, export diversification and economic growth in Egypt, Technical Paper, 135, Paris, Centre de développement de I'OCDE, juin.

Dupuch, S., Milan, C., 2003. Les déterminants des investissements directs européens dans les Pays d'Europe Centrale et Orientale, Document de travail CEPN 07-2003.

Dupuch, S., Jennequin, H, Mouhoud, E.M., 2004. EU Enlargement, what does it change for the European economic geography?, Revue de l'OFCE, numéro spécial, avril.

Dupuch, S., Jennequin, H., Mouhoud, E.M., 2001. Intégration européenne, élargissement aux PECO et économie géographique, Région et Développement 13, 125-162.

El Hédi Lahouel, M., 2001. Investissements directs étrangers, accords euro-méditerranéens et intégration entre pays du Proche-Orient et de I'Afrique du Nord, dans Dessus, S., Devlin, J., Vers une intégration régionale arabe et euro-méditerranéenne, Paris, Centre de développement de I'OCDE, 95-116.

13. Les auteurs remercient Agnès Chevallier pour ses précieux commentaires qui ont permis d'améliorer ce papier. Ils remercient également le rapporteur anonyme pour ses remarques et suggestions et restent bien entendu seuls responsables des erreurs éventuelles qui pourraient subsister. 
Emerson et al., 1990. One Market, One Money, Oxford, Oxford University Press.

Eurostat, 2002. "Investissements directs étrangers dans les pays candidats: répartition par secteur d'activité et par pays investisseur", Statistiques en bref, thème 2, 55/2002.

Finger, J.M., Kreinin, M., 1979. A measure of "export similarity" and its possible uses, The Economic Journal 89, 905-912.

Fontagné, L., Freudenberg, M., Péridy, N., 1998. Intra-industry trade and the Single Market: Quality matters, CEPR Discussion Paper 1953.

Freudenberg, M., Lemoine, F., 1999. Les pays d'Europe Centrale et Orientale dans la division du travail en Europe, Economie Internationale 80, 4 trimestre.

Hanaut, A., Loufir, R., Mouhoud, E.M., 2001. La convergence structurelle européenne: rattrapage technologique et commerce intra-branche, Économie Appliquée 1.

Kinoshita, Y., 2000. R.\&D. and technology spillovers via FDI: innovation and absorptive capacity, CERGE-El Working Paper 163.

Krugman, P., 1991. Geography and Trade, The MIT Press.

Messerlin, P., 2002. Niveau et coût du protectionnisme européen, Économie internationale 89-90, $1^{\mathrm{er}}-2^{\mathrm{e}}$ trimestre.

Midelfart-Knarvik, K.H., Overman, H.G., Redding, S.J., Venables, A.J., 2000. The location of European industry, Economic Papers 142, Commission européenne.

Milgram Baleix, J., 2001. Régionalisme versus multilatéralisme: le cas du Maghreb, Thèse de doctorat en sciences économiques, Université Paris I Panthéon Sorbonne.

Mouhoud, E.M., 1998. Investissements directs internationaux et intégration régionale dans les pays du Bassin méditerranéen, dans Migrations, libre-échange et intégration régionale dans le Bassin méditerranéen, OCDE, Paris.

Rutherford, T.F,. Rutstrom, E.E., Tarr, D., 1993. Morocco's Free Trade Agreement with the European community, Policy Research Working Paper 1173, Banque mondiale, Washington, D.C.

Talahite, F. 1996. Le partenariat euro-méditerranéen vu du Sud, Monde Arabe, Maghreb-Machrek 153, Paris, La Documentation française, 45-60. 
